\title{
Modeling of Radiation Effects in the MIS Devices
}

\author{
Galina Zayats ${ }^{1}$, Alexandr Komarov ${ }^{2}$, Fadei Komarov ${ }^{2}$, Sergei Miskiewicz ${ }^{2}$ \\ ${ }^{1}$ Institute of Mathematics, Academy of Sciences of Belarus, Minsk, Belarus \\ ${ }^{2}$ Institute of Applied Physics Problems, Minsk, Belarus
}

Email address:

zayats@im.bas-net.by (G. Zayats), komarovf@bsu.by (A. Komarov)

\section{To cite this article:}

Galina Zayats, Alexandr Komarov, Fadei Komarov, Sergei Miskiewicz. Modeling of Radiation Effects in the MIS Devices. American Journal of Nano Research and Applications. Vol. 5, No. 1, 2017, pp. 7-11. doi: 10.11648/j.nano.20170501.12

Received: March 9, 2017; Accepted: March 28, 2017; Published: April 19, 2017

\begin{abstract}
We describe space-time evolution of electric charge induced in dielectric layer of simulated metal-insulatorsemiconductor structures produced by irradiation with X-rays. The purpose of this article is to develop a model which most fully describes the phenomena occurring under the ionizing irradiation of MOS structures. For this, in addition to known processes, such as a generation of the electron-hole pairs in the dielectric volume, diffusion and drift in the electric field of mobile charge carriers (electrons and holes), capture holes at the traps and recombination of electrons with the trapped holes, we also took into account the formation of surface states. The mathematical model considered includes the continuity equations for free electrons and holes, the Poisson equation, the equation describing the kinetics of hole-charge accumulation at trap levels, and the equation describing the tunneling mechanism of discharge of the charge accumulated in the dielectric. The model developed makes it possible to simulate the processes of charge degradation of silicon structures by the effect of ionizing radiation, and to determine the change in the threshold voltage of the MIS structure under irradiation, the distribution of free and trapped charges in the dielectric, and the distribution of the electric field strength. The type of dose dependence of the change in the threshold voltage of the MIS structure is determined by a number of parameters: the concentration of the traps in the oxide, their distribution over the oxide thickness, the mobility and capture cross sections for electrons and holes, the nature of dependence of these parameters on the electric field in the oxide. The system of equations is solved iteratively by efficient numerical method. The obtained simulation results are in good agreement with the corresponding data presented in other scientific publications.
\end{abstract}

Keywords: Ionizing Irradiation, Space Charge, Surface States, Numerical Simulation, MIS

\section{Introduction}

The problem of forecasting and control of bipolar and CMOS ICs radiation resistance is of great importance today. Currently, integrated circuits (IC) are essential part of military and space equipment. As being used in space, such equipment is inevitably exposed to low-level ionizing radiation, causing its degradation and finally malfunction. Therefore, one of the urgent tasks of the microelectronics is to design and manufacture ICs with much higher radiation resistance. In this context, mathematical modeling has paramount importance, by providing solid ground for both understanding and prediction of radiation effects of X-rays in semiconductor devices. Such a simulation necessarily includes development of efficient diffusion-kinetic models and computational algorithms.

The effect of ionizing radiation on MOS structures was studied and modeled in a number of investigations [1-13]. At first, these were simplified models in which the distribution of the space charge caused by ionizing radiation was given in the form of some dependences at a number of constraints [13]. Later, there were developed models fully taking into account physical phenomena occurring in MOS structures under irradiation. Thanks to numerical methods, it became possible to investigate models of any complexity. Thus, in [4], the distributions of the potential, the field strength, the concentration of free and bound charge carriers over the depth of the dielectric under irradiation of the MOS structure as a function of the dose and voltage on the gate were obtained, and the parameters of the model were determined that give the best correspondence with the experimental dependences. In the model of Gurtov et al. [5], the possibility of tunneling carriers trapped in high fields was taken into account. In the model of Levin and co-authors [6], along with 
the process of accumulation of an induced charge in a dielectric, the subsequent charge relaxation due to tunneling discharge and thermal emission from shallow and deep traps was considered. Attempts of the quantitative consideration of the formation of surface states at the interface between semiconductor silicon and oxide were made in [1, 2, 7-9].

We considered the $\mathrm{SiO}_{2} / \mathrm{Si}$ part of a simulated metal-oxidesemiconductor (MOS) structure with two types of trap levels that takes into account both defects within the oxide layer and radiation-induced interface states. In this way we developed both physical and mathematical models of radiation-induced charge accumulation within the oxide layer and surface states due to irradiation with X-rays and the subsequent charge relaxation by means of tunnel discharge. Developed mathematical model is the system of equations [6, 14] describing the free electron and hole kinetics, the kinetics of hole charge accumulated on the shallow and deep trap levels and the electric field intensity in dielectric layer. The tunnel discharge of the captured hole charge in the dielectric layer is described in [15]. Potential distribution in MOS structure in the presence of charge in dielectric layer and on the surface states is given by electro neutrality.

The numerical solution is based on the difference method [16]. The developed iterative algorithm allowed us to simulate the following properties of the MOS structure: radiation-induced changes of the threshold voltage as a function of radiation dose, electric charge distribution in oxide layer with various thickness, the resulting effective charge and electric field within the MOS structure during irradiation, etc.

\section{The Model}

The following system of equations describes the radiation dynamics of electric charge distribution in the dielectric layer with thickness $d$ of MIS [6-14, 15]. These equations take into account tunnelling discharge as well.

$$
\begin{aligned}
& \frac{\partial n}{\partial t}=D_{n} \frac{\partial^{2} n}{\partial x^{2}}+\mu_{n} \frac{\partial(n \cdot E)}{\partial x}- \\
& R_{n 1}\left(n, E, P_{t 1}\right)-R_{n 2}\left(n, E, P_{t 2}\right)+G(E) \\
& \frac{\partial p}{\partial t}=D_{p} \frac{\partial^{2} p}{\partial x^{2}}+\mu_{p} \frac{\partial(p \cdot E)}{\partial x}- \\
& R_{p 1}\left(p, E, P_{t 1}\right)-R_{p 2}\left(p, E, P_{t 2}\right)+G(E) \\
& \frac{\partial P_{t 1}}{\partial t}=R_{p 1}\left(p, E, P_{t 1}\right)-R_{n 1}\left(n, E, P_{t 1}\right) \\
& \frac{\partial P_{t 2}}{\partial t}=R_{p 2}\left(p, E, P_{t 2}\right)-R_{n 2}\left(n, E, P_{t 2}\right) \\
& \frac{\partial E}{\partial x}=\frac{q}{\varepsilon_{0 x} \varepsilon_{0}}\left(P_{t 1}+P_{t 2}+p-n\right) \\
& \frac{\partial P_{t}}{\partial t}=-\alpha_{1} \exp \left(-\alpha_{2} x\right) P_{t}
\end{aligned}
$$

$$
V_{G}=\phi_{m s}+\psi-\left(\frac{Q_{0 t}}{C_{o x}}+\frac{Q_{s c}(\psi)}{C_{o x}}+\frac{Q_{s s}(\psi)}{C_{o x}}\right)
$$

The respective initial and boundary conditions are as follows:

$$
\begin{aligned}
& n(0, t)=n(d, t)=0, p(0, t)=p(d, t)=0, \\
& 0<t \leq t_{f} ; Q_{0 t}(0)=Q_{s s}(0)=0 ; \\
& n(x, 0)=p(x, 0)=P_{t 1}(x, 0)=P_{t 2}(x, 0)=0, \\
& E(x, 0)=f(\psi(0)), \quad 0 \leq x \leq d .
\end{aligned}
$$

The equations (1)-(7) contain the following parameters: $t_{f}$ is a simulation time, $n, p$ stand for concentration of free electrons and holes, $E$ is electric field, $P_{t 1}$ is concentration of holes captured on the shallow trap levels (both oxide-gate and oxide-semiconductor interfaces), $P_{t 2}$ is concentration of holes captured on the deep trap levels (inside the oxide layer), $D_{n}, D_{p}$ are diffusion coefficients of electrons and holes, $\mu_{n}, \mu_{p}$ are mobility of electrons and holes, $G$ is generation rate of the electron-hole pairs due to ionizing radiation, $q$ is electron charge, $\varepsilon$ is dielectric permittivity of $\mathrm{SiO}_{2}, R_{n 1,2}, R_{p 1,2}$ are capture rates of electrons and holes on the shallow and deep trap levels, $V_{G}$ is gate voltage, $\varphi_{m s}$ is difference of the work functions of the gate and semiconductor materials, $\psi$ is surface potential of semiconductor, $\alpha_{1}, \alpha_{2}$ are frequency and barrier factors, $C_{o x}$ is dielectric layer capacity, $Q_{s s}$ is surface state charge, $Q_{s c}$ is charge of the space-charge region of the semiconductor, $Q_{0 t}$ is the effective charge captured on the trap levels in $\mathrm{SiO}_{2}$ layer.

The model is complemented by the following equations. Capture rates of electrons and holes on the trap levels are defined as in ref. [6]

$$
\begin{gathered}
R_{n 1}=n P_{t 1} \sigma_{n}(E)\left(\mu_{n}|E|+v_{t h}\right) \\
R_{n 2}=n P_{t 2} \sigma_{n}(E)\left(\mu_{n}|E|+v_{t h}\right) \\
R_{p 1}=p\left(N_{t 1}-P_{t 1}\right) \sigma_{p}(E)\left(\mu_{p}|E|+\frac{\mu_{p}}{\mu_{n}} v_{t h}\right) \\
R_{p 2}=p\left(N_{t 2}-P_{t 2}\right) \sigma_{p}(E)\left(\mu_{p}|E|+\frac{\mu_{p}}{\mu_{n}} v_{t h}\right)
\end{gathered}
$$

where $N_{t 1,2}(x)$ are concentrations of hole traps; $v_{t h}$ is the thermal velocity of charge carriers; $\sigma_{p}(E)$ and $\sigma_{n}(E)$ are capture cross-sections for holes and electrons, respectively.

The generation rate of electron-hole pairs $\mathrm{G}(E)$ depends on radiation dose intensity $\dot{D}=d D / d t$, pairs generation coefficient $k_{g}$, and the probability for the created electronhole pairs to be separated by electric field before 
recombination $f_{y}^{x-r a y}(E)[17,18]: G(E)=\dot{D} k_{g} f_{y}^{x-r a y}(E)$.

Charge of the space-charge region, the electric charge in the bulk of the oxide layer and at the interfaces are calculated as

$$
\begin{gathered}
Q_{s c}(\psi)=\varepsilon_{s} \varepsilon_{0} E_{s}= \pm \frac{\sqrt{2} \varepsilon_{s} \varepsilon_{0} k T}{q L_{D}} F\left(\psi, \varphi_{0}\right) \\
Q_{0 t}=\frac{1}{d} \int_{0}^{d}(d-x) \rho_{t}(x) d x \\
Q_{s s}=q N_{s s}\left(\varphi_{0}-\psi\right)
\end{gathered}
$$

where $\rho_{t}(x)$ is distribution of hole charge accumulated on the trap levels, $N_{s s}=k_{D} \frac{Q_{0 t}}{q \varphi_{0}}$ is the surface state density [19, 20] averaged to the band gap energy ( $k_{D}$ is determined experimentally).

\section{The Modified System of Equations}

Taking into account Eq. (8), the system (1) - (6) can be written in the following form suitable for constructing the solution algorithm:

$$
\begin{gathered}
\frac{\partial n}{\partial t}=D_{n} \frac{\partial^{2} n}{\partial x^{2}}+\mu_{n} \frac{\partial(n \cdot E)}{\partial x}-n Q_{1}\left(E, P_{t 1}, P_{t 2}\right)+G(E) \\
\frac{\partial p}{\partial t}=D_{p} \frac{\partial^{2} p}{\partial x^{2}}-\mu_{p} \frac{\partial(p \cdot E)}{\partial x}-p Q_{2}\left(E, P_{t 1}, P_{t 2}\right)+G(E) \\
\frac{\partial P_{t 1}}{\partial t}=-P_{t 1} S_{1}(p, n, E)+S_{2}(p, E) N_{t 1} \\
\frac{\partial P_{t 2}}{\partial t}=-P_{t 2} S_{1}(p, n, E)+S_{2}(p, E) N_{t 2} \\
\frac{\partial E}{\partial x}=\frac{q}{\varepsilon_{0 x} \varepsilon_{0}}\left(P_{t 1}+P_{t 2}+p-n\right) \\
\frac{\partial P_{t}}{\partial t}=-P_{t} S_{3}(E) \\
-V_{G}+\phi_{m s}+\psi-\left(\frac{Q_{0 t}}{C_{o x}}+\frac{Q_{s c}(\psi)}{C_{o x}}+\frac{Q_{s s}(\psi)}{C_{o x}}\right)=0
\end{gathered}
$$

Where

$$
\begin{gathered}
Q_{1}\left(E, P_{t 1}, P_{t 2}\right)=\left(P_{t 1}+P_{t 2}\right) \sigma_{n}(E)\left(\mu_{n}|E|+v_{t h}\right) \\
Q_{2}\left(E, P_{t 1}, P_{t 2}\right)=\left(N_{t 1}-P_{t 1}+N_{t 2}-P_{t 2}\right) \sigma_{p}(E)\left(\mu_{n}|E|+\frac{\mu_{\mathrm{p}}}{\mu_{\mathrm{n}}} v_{t h}\right)
\end{gathered}
$$

$$
\begin{gathered}
S_{1}(p, n, E)=p \sigma_{p}(E)\left(\mu_{p}|E|+\frac{\mu_{p}}{\mu_{n}} v_{t h}\right)+n \sigma_{n}\left(\mu_{n}|E|+v_{t h}\right) \\
S_{2}(p, E)=p \sigma_{p}(E)\left(\mu_{p}|E|+\frac{\mu_{p}}{\mu_{n}} v_{t h}\right) \\
S_{3}(E)=\alpha_{1}(E) \exp \left(-\alpha_{2} x\right)
\end{gathered}
$$

\section{The Solution Algorithm}

The solution of the system of equations (11)-(16) with boundary conditions (7) is based on iteration algorithm. Each time step $t=t_{j}$ includes the following stages:

(a) First, the initial-boundary value problem for the continuity equation (11) together with the initial and boundary conditions (7) is solved in order to calculate the depth distribution of free electrons $n\left(x, t_{j}\right)$.

(b) Next, by taking into account the results obtained at the previous stage and by solving the initial-boundary value problem for the continuity equation (12) one can calculate the depth distribution of holes $p\left(x, t_{j}\right)$.

(c) Further, the calculated distributions of free charge carriers $n\left(x, t_{j}\right)$ and $p\left(x, t_{j}\right)$ are put into the equation (13) describing capture kinetics of holes. In this way the spatial distribution of hole charge accumulated on the trap levels $P_{t 1}\left(x, t_{j}\right)$ and $P_{t 2}\left(x, t_{j}\right)$ is determined.

(d) Then the equation (15) is solved in order to take into account the tunnel discharging of captured charge in dielectric layer.

(e) Next, the depth distributions of free electrons $n\left(x, t_{j}\right)$, free holes $p\left(x, t_{j}\right)$, and captured charge $P_{t 1}\left(x, t_{j}\right)$ and $P_{t 2}\left(x, t_{j}\right)$ found at previous stages are put into the Poisson equation (14) thus allowing to compute the electric field in the oxide layer.

(f) Using the results of all the previous stages, one can solve the equation of electro-neutrality (16) defining many properties of the MOS structure as a whole and calculate the value of the surface potential of semiconductor $\psi\left(t_{j}\right)=\stackrel{s+1}{\psi}$, where $s=0,1,2 \ldots$ is the iteration number.

(g) The termination condition for time step $t=t_{j}$ is $\left|\begin{array}{cc}s+1 & s \\ \psi-\psi\end{array}\right| \leq \varepsilon_{1}\left|\begin{array}{c}s \\ \psi\end{array}\right|+\varepsilon_{2}, \varepsilon_{1}, \varepsilon_{2}$ are the empirical constants $\left(\varepsilon_{1}=0\right.$, if $\left|\begin{array}{l}\psi \\ \psi\end{array}\right|<1$ and $\varepsilon_{2}=0$, if $\left|\begin{array}{c}s \\ \psi\end{array}\right|>1$ ). If the indicated termination condition is fulfilled, we calculate the threshold voltage, the effective charge in oxide layer and other characteristics of MOS-structure at time $t_{j}$. Otherwise, we first use the obtained distribution $E\left(x, t_{j}\right)$ to recalculate the functions $n\left(x, t_{j}\right), p\left(x, t_{j}\right), P_{t 1}\left(x, t_{j}\right)$ and $P_{t 2}\left(x, t_{j}\right)$. Finally, we 
increment time and thus proceed to the next time step going again through stages (a)-(g) mentioned above.

The presented algorithm is applied numerically. Developed numerical method is based on the difference method [16]. The difference problem is solved iteratively. The numerical solution of the initial-boundary problems for the continuity equations $n\left(x, t_{j}\right), p\left(x, t_{j}\right)$ is obtained by the sweep method [16]. The electro neutrality equation (16) is solved by means of bisection method.

\section{Results}

The numerical simulations of radiation-induced changes in the threshold voltage of the MOS structures due to $20 \mathrm{keV} \mathrm{X-}$ rays have been performed. Some results of simulation are presented in Figures 1-3. The calculations were done with the following values: the radiation dose of X-rays $D=5 \times 10^{5}$ R with intensity $\dot{D}=d D / d t=10^{2} \mathrm{R} / \mathrm{s}$, the boron concentration in the semiconductor $N_{B}=10^{15} \mathrm{~cm}^{-3}$, temperature $T=300 \mathrm{~K}, \varphi_{m s}=-0.5 \mathrm{~V}$, the mobility of electrons $\mu_{n}=10^{2} \mathrm{~cm}^{2} \mathrm{~V}^{-1} \mathrm{~s}^{-1}$ and holes $\mu_{p}=0,6 \times 10^{-3} \mathrm{~cm}^{2} \mathrm{~V}^{-1} \mathrm{~s}^{-1}$ in oxide layer. The generation rate of electron-hole pairs in $\mathrm{SiO}_{2}$ is $k_{g}=8 \times 10^{12} \mathrm{~cm}^{-3} \mathrm{rad}^{-1}$ pairs, the permittivity values are $\varepsilon_{o x}=1.6$ and $\varepsilon_{s}=11.5, V_{G}=-1 V, k_{D}=1.15$.

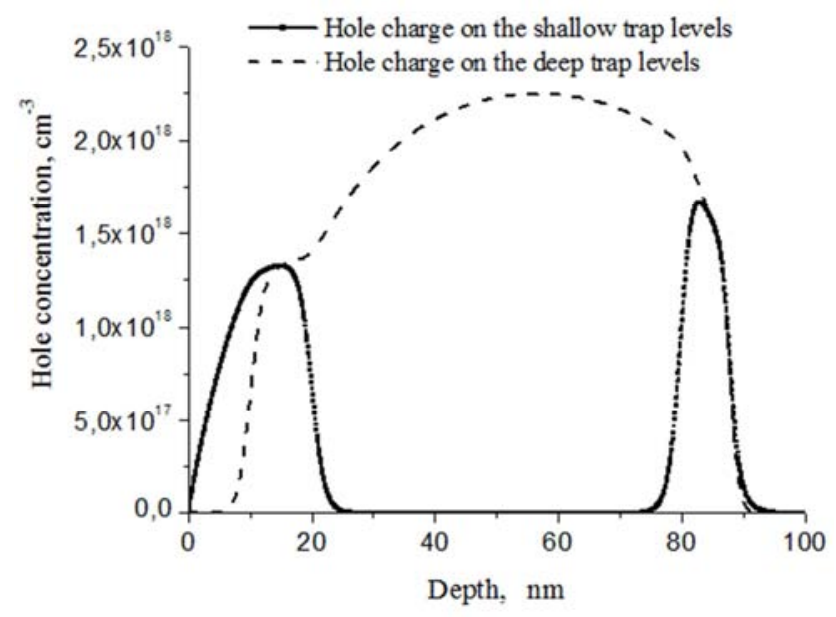

Figure 1. Depth distribution of holes on the trap levels after the irradiation.

Figure 1 shows the calculated depth distribution of holes bound by both "shallow" and "deep" trap levels in $\mathrm{SiO}_{2}$ layer thickness $d$ of $100 \mathrm{~nm}$. The calculations were made for $V_{G}=-0.9 \mathrm{~V}$ and the distribution of "shallow" $N_{t 1}$ and "deep" $N_{t 2}$ trap levels [6]

$$
\begin{aligned}
& N_{t 1}=5 \times 10^{18}\left(\left(e^{(x-20)}+1\right)^{-1}+\left(e^{(80-x)}+1\right)^{-1}\right) \\
& N_{t 2}=5 \times 10^{18}\left(\left(e^{(x-90)}+1\right)^{-1}-\left(e^{(x-10)}+1\right)^{-1}\right)
\end{aligned}
$$

Figure 2 illustrates the dependence of the radiationinduced changes of the threshold voltage of the MOS structure on the ionizing radiation dose for the different concentrations of the "deep" trap levels $\mathrm{N}_{\mathrm{t} 2}$ (the concentration of the "shallow" trap levels $N_{t 1}=10^{18} \mathrm{~cm}^{-3}$ ). Figure 3 illustrates depth distribution of holes bound on "shallow" and "deep" trap levels in $\mathrm{SiO}_{2}$ for various thickness of the oxide layer. One can see that the thinner the oxide layer, the lower is concentration of accumulated hole charge distribution on the "deep" and "shallow" trap levels. Consequently, MOS structures with a thin oxide layer have greater radiation resistance than structures having a thicker dielectric layer. The obtained simulation results correlate well with data in Refs. [6, 21].

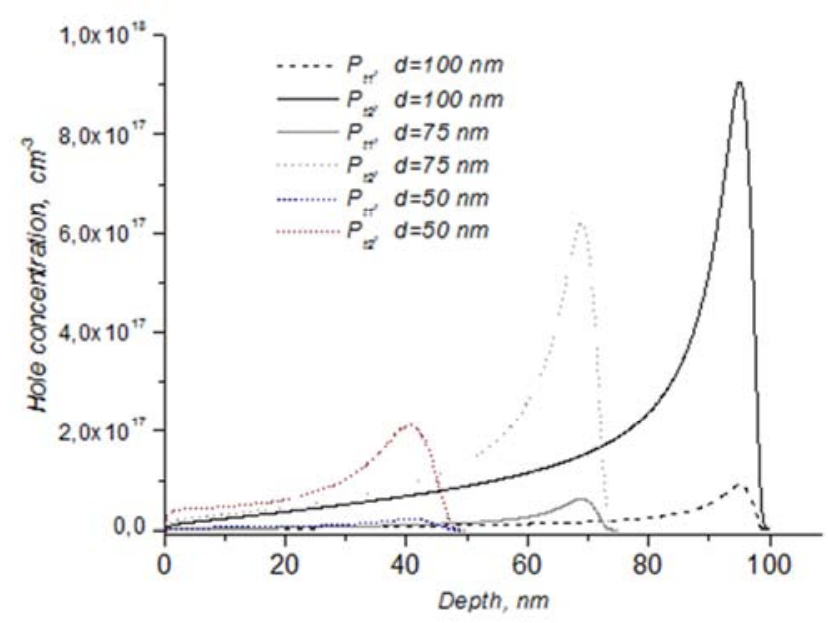

Figure 2. Dependence of the radiation-induced changes of the threshold voltage of the MOS structure on the ionizing radiation dose for the different concentrations of the "deep" trap levels $N_{t 2}$.

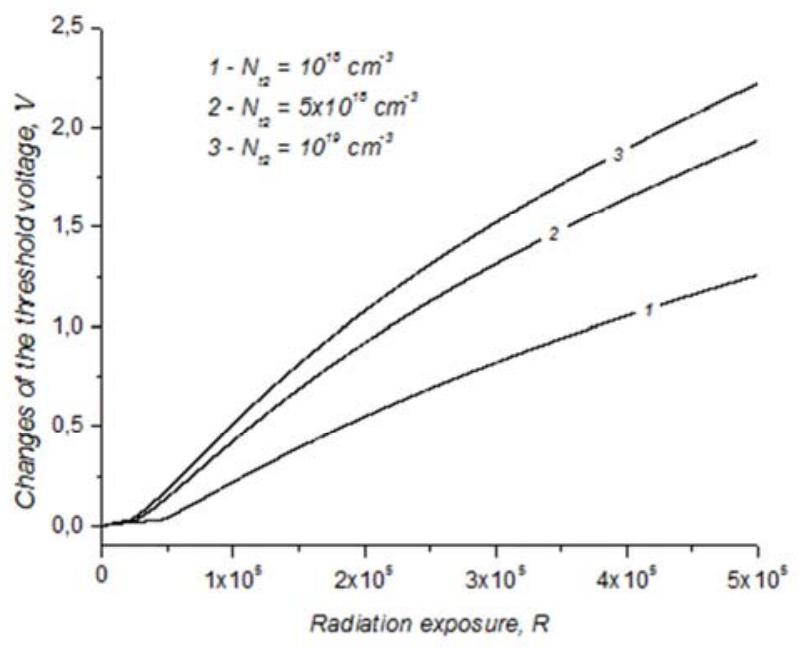

Figure 3. Hole charge distribution on the "deep" and "shallow" trap levels for the different values of dielectric layer thickness. The concentration of "deep" trap levels $N_{t 2}=10^{19} \mathrm{~cm}^{-3}$ and "shallow" trap levels $N_{t 2}=10^{18} \mathrm{~cm}^{-3}$.

\section{Conclusions}

The offered model can be used to simulate radiationinduced deterioration of MOS structures and to calculate the radiation-induced changes in the MOS threshold voltage depending on the depth distribution of the traps within the 
silicon oxide layer and on the mobility and capture crosssections of electrons and holes. Furthermore, also depth distributions of free and bound/trapped electrons and holes in $\mathrm{SiO}_{2}$ layer, the resulting electric field intensity, and the change of surface potential of the oxide-semiconductor interface in MOS structure can be computed as well.

\section{References}

[1] F. P. Korshunov, Yu. V. Bogatyrev, and V. A. Vavilov, Effect of Radiation on Integrated Circuits. Nauka Tekhnika, Minsk, 1986. [in Russian].

[2] V. S. Pershenkov, V. D. Popov, and A. V. Shal'nov, Surface Radiation Effects in Integrated Circuits. Energoatomizdat, Moscow, 1988. [in Russian].

[3] J. P. Mitchell, "Radiation-Induced Space-Charge Buildup in MOS Structures", IEEE Trans. Electron Dev. 1967. Vol. 14, no. 11. P. 764-774.

[4] J. N. Churchill, F. E. Holstrom, and T. W. Collins, "Dynamic model for e-beam irradiation of MOS capacitors", J. Appl. Phys. 1979. Vol. 50, № 6. P. 3094-4002.

[5] V. A. Gyrtov, A. N. Nazarov, N. Travkov, "Modeling of the process of space charge accumulation in MIS insulators of structures upon irradiation", Fizika I technika poluprovodnikov [Semiconductors]. 1990, Vol. 24, no. 6. P. 969-977.

[6] M. N. Levin, A. V. Tatarintsev, V. A. Makarenko V. A., V. R. Gitlin, "X-ray or UV adjustment of MOS threshold voltage: Analytical and numerical modeling", Russ. Microelectronics. 2006, Volume 35, Issue 5, pp 329-336.

[7] D. A. Buchanan, "On the generation of interface states from electron-hole recombination in metaloxidesemi-conductor capacitors", Appl. Phys. Lett. 1994. Vol. 65, №10. P. $1257-$ 1259 .

[8] O. V. Aleksandrov, S. A. Visotskaya, V. S. Zhurkin, "Model of charge of accumulation in MOS-transistors at ionizing irradiation", Izvestiya St. Petersburg State Electrotechnical University "LETI". 2012, no. 7. P. 20-27.

[9] O. V. Aleksandrov, "Model of the behavior of MOS structures under ionizing irradiation", Semiconductors. 2014. Vol. 48, issue 4. P. 505-510.
[10] V. Rusanovschi, A. Avram, "Numeric modeling and analytical solution of ionizing irradiation induced charge in MOSFET structure oxide". In: ISETS'14. The conference proceedings of 11th International Symposium on Electronics and Telecomunications, Timisoara, Romania. Timişoara: IEEE, 2014, pp. 29-32.

[11] C. Claeys, E. Simoen, Radiation Effects in Advanced Semiconductor Materials and Devices. Berlin, 2002.

[12] Yu. V. Bogatyrev, S. B. Lastovsky, S. A. Soroka, S. V. Shwedov, and D. A. Ogorodnikov, "Influence of gamma radiation on MOS/SOI transistors", Reports of BGUIR. 2016. no. 3 (97). P. $75-80$.

[13] H. E. Boesch, F. B. McLean, J. M. Benedetto, and J. M. McGarrity, "Saturation of Threshold Voltage Shift in MOSFET's at High Total Dose", IEEE Trans. Nucl. Sci. 1986, vol. 33, no. 6, pp. 1191-1197.

[14] G. M. Zayats, F. F. Komarov, A. F. Komarov, S. A. Miskiewicz, "Simulation of inffluence of low-intensity space ionizing radiation on the MIS charge state". Reports of NAS of Belarus. 2013. V. 57, no. 3. Pp. 53-57. [in Russian].

[15] P. J. MCWhorter, S. L. Miller, W. M. Miller, "Modeling the anneal of radiation-induced trapped holes in a varying thermal environmen". IEEE Trans. Nuclear Physics. 1990. V. 37, no. 6. Pp. $1682-1689$.

[16] A. A. Samarskij, Theory of Difference Schemes. Nauka, Moscow, 1989. [in Russian].

[17] G. A. Ausman, F. B. McLean, "Electron-Hole Pair Creation Energy in $\mathrm{SiO}_{2}$ ”. Appl. Phys. Lett. 1975. V. 26. Pp. 173.

[18] J. M. Benedetto, and H. E. Boesch, "The Relationship between $\mathrm{Co}_{60}$ and $10 \mathrm{-keV}$ X-ray Damage in MOS Devices", IEEE Trans. Nucl. Sci. 1986, vol. 33, no. 6, pp. 1318-1323.

[19] A. G. Kadmenskij, S. G. Kadmenskij, M. N. Levin, V. M. Maslovskij, V. E. Chernyshev, JETP Lett. 1993. V. 19. Pp. 4145 .

[20] M. N. Levin, and V. M. Maslovsky, "Relaxation Processes Induced in $\mathrm{Si}_{-} \mathrm{SiO}_{2}$ Systems by Ionizing Radiation and Pulsed Magnetic Field Treating”, Solid State Commun., 1994, vol. 90, no. 12 , pp. 813-816.

[21] M. N. Levin, A. V. Tatarintzev, E. V. Bondarenko, A. E. Bormontov, V. R. Gitlin, "Prediction the radiation resistance of MOS IS in conditions of low-intensity irradiation". Condenced matters and phase interfaces. 2010. V. 12, no. 3. Pp. 226-232. 